\title{
Selfconsistent Models of Winds from Rotating Early-Type Stars - Application to B[e] star winds
}

\author{
P. Petrenz \& J. Puls \\ Institute for Astronomy and Astrophysics of the \\ Ludwig-Maximilians-University, Scheinerstr. 1, D - 81679 Munich
}

\begin{abstract}
We present results of 2.5-D radiation hydrodynamical simulations of winds from rapidly rotating stars. For the first time, we consider the dependence of the line statistics on local density and radiation temperature implying a spatial variation of the force-multiplier parameters $k, \alpha$ and $\delta$ which control the dynamics of the flow.

We apply our models to the problem of disk formation in $\mathrm{B}[\mathrm{e}]$-star winds.
\end{abstract}

\section{Winds from rotating early-type stars}

In recent years, the actual structure of radiation driven winds from rotating early-type stars has been discussed without resolution. Bjorkman \& Cassinelli (1993) dropped for the first time the assumption of spherical symmetry and solved for the particle trajectories in the polar plane, thus adopting only azimuthal symmetry about the rotational axis. In the equations of motion, they considered additionally to the inertial accelerations a purely radial line force calculated within the force-multiplier concept, where the corresponding forcemultiplier parameters (fmps) $k_{\mathrm{CAK}}, \alpha, \delta$ (cf. Castor et al. 1975, Abbott 1982) were assumed to be constant throughout the wind, i.e., independent both of distance $r$ and co-latitude $\Theta$.

This model predicts a polar deflection of the wind material towards the equatorial plane leading to the formation of a wind-compressed disk (WCD) in the equatorial plane for extreme rotation rates $\Omega \gtrsim 0.9(0.5)$ for $\mathrm{O}$-(B-)stars (for a confirmation by radiation hydrodynamical simulations, see Owocki et al. (1994).

Owocki et al. (1996) additionally accounted for non-radial line force components $g_{\Theta}^{\text {lines }}$ and $g_{\Phi}^{\text {lines }}$ (acting in the polar and azimuthal direction, respectively) and gravity darkening.

The negative $g_{\Theta}^{\text {lines }}$ inhibits the disk formation and redistributes the wind material towards the poles, and the negative $g_{\Phi}^{\text {lines }}$ spins down the wind (by up to $\lesssim 35 \%$ of the value given by angular momentum conservation).

The gravity darkening implies an enhanced (reduced) photon flux over the poles (in the equatorial plane) and thus leads, in combination with the non-radial line force components, to an unambigously prolate wind, which is dense and fast over the poles and slower and thinner in the equatorial plane.

These results are entirely contradictory to the predictions by the simple $\mathrm{BC}$ model and, meanwhile, have been independently confirmed by Petrenz (1999).

However, also these investigations suffer from the assumption of $k_{\mathrm{CAK}}, \alpha$, $\delta$ constant throughout the wind, which have been adopted from 1-D non-LTE 
calculations and, in the 2-D case, should be affected by at least two processes. Firstly, both density and ionizing radiation field are a function of co-latitude $\Theta$, and secondly, the assumption of constant fmps as function of $r$ is rather questionable even for a uniformly bright stellar surface (cf. Kudritzki et al. 1998). Thus, these global 1-D parameters do not correctly account for the influence of local physical conditions (density, velocity field) and the non-local stellar radiation field on ionization stratification and occupation numbers (line opacities) in the wind.

Therefore, we have dropped this approximation and developed a more realistic 2-D non-LTE description which provides fmps that are consistent both with the local hydrodynamic properties of the flow and the non-local latitudedependent radiation field (for further details, see Petrenz \& Puls (1999).

\section{The models}

The basic issue of our study was to model the actual morphology of radiation driven winds from rapidly rotating stars, which is essential for the subsequent application of theoretical models to spectrum synthesis, e.g., in the case of the $\mathrm{H}_{\alpha}$ emission line in winds from O-supergiants (Petrenz \& Puls 1996).

We have to discriminate between winds with an optically thin continuum and winds where the mass-loss exceeds a critical value and the continuum may become optically thick.

Optically thin continuum. The optical depth in the Lyman continuum $\tau_{L y}$ is less than unity at every co-latitude for a B-star with $T_{\text {eff }}=20000 \mathrm{~K}, r_{*}=30 \mathrm{R}_{\odot}$, $M_{*}=30 \mathrm{M}_{\odot}$ and $v_{\text {rot }}=0.85 v_{\text {crit }}=290 \mathrm{~km} \mathrm{~s}^{-1}$. Therefore, the assumption of an optically thin continuum is justified. (Eq. 7 from Lamers and Pauldrach (1991, hereafter LP91) yields $\tau_{\mathrm{Ly}}=0.42$ at the pole and $\tau_{\mathrm{Ly}}=0.04$ at the equator.)

Fig. 1 displays the density structure and the radial velocity field of this wind model after a flow time of $2 \cdot 10^{6} \mathrm{~s}$.

A clearly prolate wind structure shows up, with a polar density contrast, which significantly increases for larger radii $r$ with maximum values $\rho_{\text {pol }} / \rho_{\text {eq }} \approx$ 15. The wind is fast over the poles, with a final velocity $v_{\infty}\left(0^{\circ}\right) \approx 1030 \mathrm{~km} \mathrm{~s}^{-1}$, and slowest in the equatorial plane, with $v_{\infty}\left(90^{\circ}\right) \approx 730 \mathrm{~km} \mathrm{~s}^{-1}$. Owing to the negative polar component $g_{\Theta}^{\text {lines }}$, which arises from local asymmetries of the projected velocity gradients, the matter is deflected towards the polar regions with minimum polar velocities $v_{\Theta, \min } \approx-50 \mathrm{~km} \mathrm{~s}^{-1}$. As we see, also a selfconsistent description of the wind dynamics does not predict the formation of an equatorial disk, but rather suggests the contrary effect!

This morphology can be understood by considering the contribution from various elements to the total line acceleration both over the poles and in the equatorial plane, as displayed in Fig. 2 (top), where we have plotted the logarithm of the quantity

$$
N_{\text {eff }}=\sum_{\text {lines L }} \frac{\nu F_{\nu}}{F}\left(1-e^{-\tau_{\mathrm{s}}}\right)
$$

which may be regarded as the effective number of optically thick lines (cf. Abbott 1980). Iron clearly dominates throughout the wind in both cases, owing to 

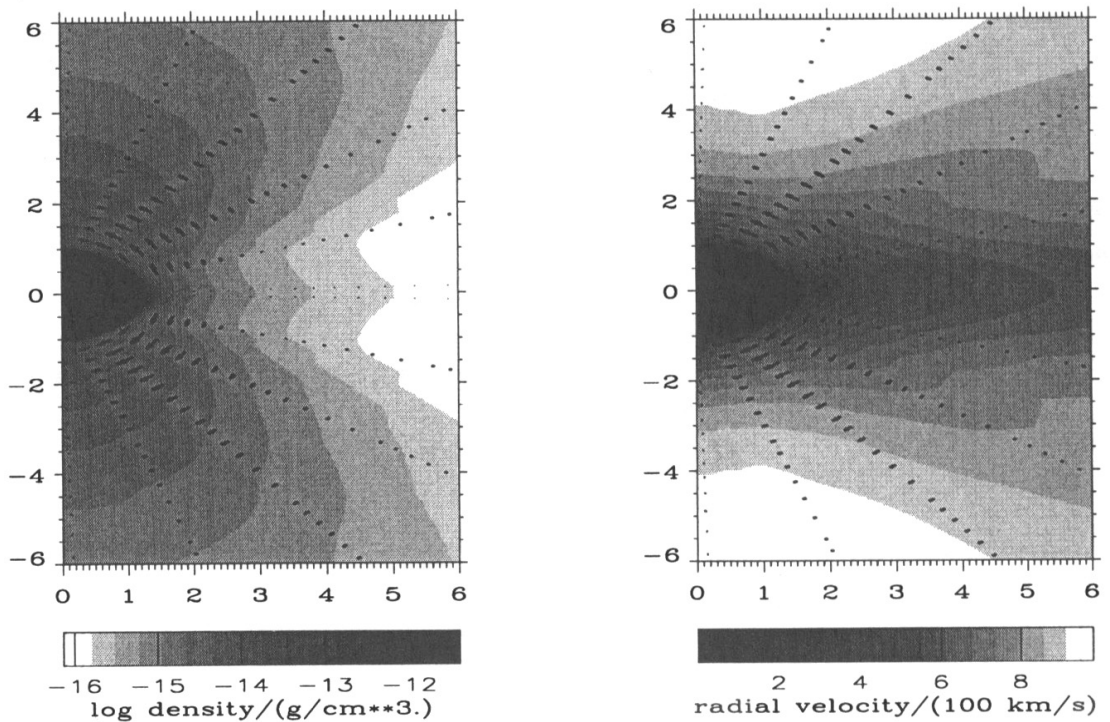

Figure 1. Density and radial velocity component of a B-star wind with $T_{\text {eff }}=20000 \mathrm{~K}, r_{*}=30 \mathrm{R}_{\odot}, M_{*}=30 \mathrm{M}_{\odot}, v_{\text {rot }}=0.85 v_{\text {crit }}=$ $290 \mathrm{~km} \mathrm{~s}^{-1}$ and consistent fmps. The arrows indicate the polar velocities with a minimum value $v_{\Theta, \min } \approx-50 \mathrm{~km} \mathrm{~s}^{-1}$.
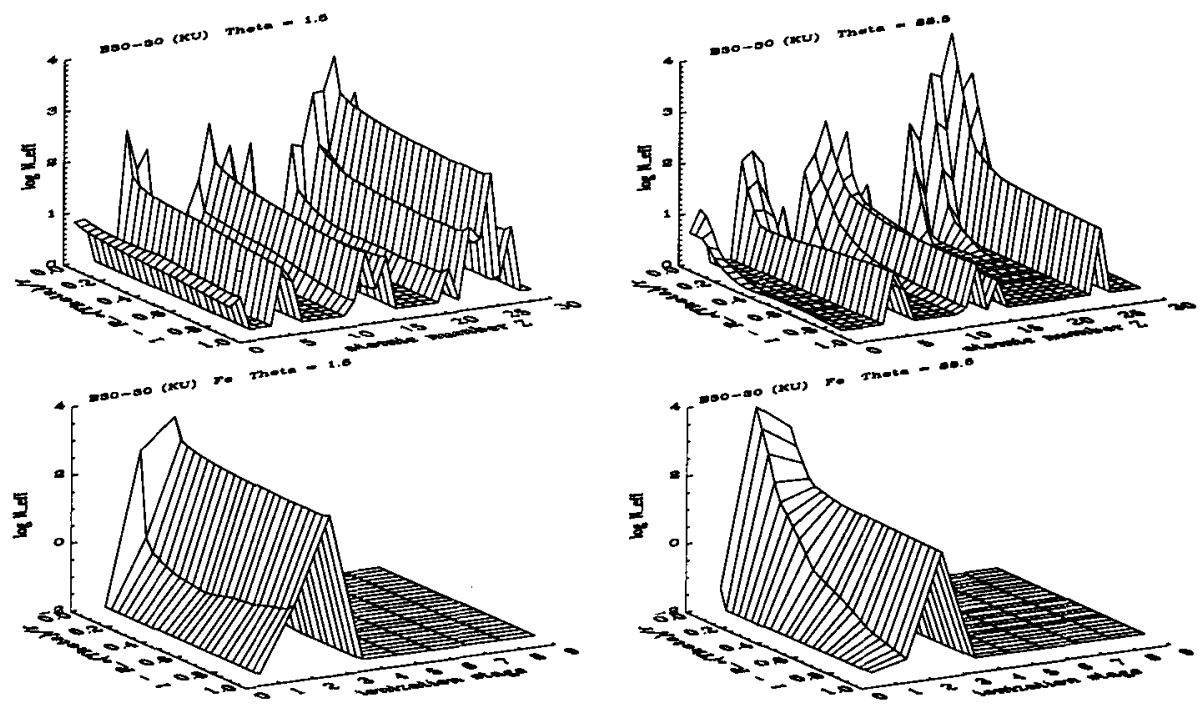

Figure 2. Top: $N_{\text {eff }}$ for the B star wind (optically thin continuum) over atomic number $Z$ and radius, over the pole (left) and in the equatorial plane (right). Bottom: $N_{\text {eff }}$ for the various ionization stages of iron over the pole (left) and in the equatorial plane (right). 
hundreds of thousands of (unsaturated) metastable lines. According to Fig. 2 (bottom), the main contribution originates from Fell, which is the major ionization stage in the entire wind.

Since there are no abrupt polar ionization gradients in the wind, which might give rise to a significant polar variation of $\dot{M}$ (if lower ionization stages with more driving lines would dominate the ionization equlibrium), the prolate wind morphology arises from the temperature dependence of the line force, in a similar way as in the wind models with constant $k_{\mathrm{CAK}}, \alpha, \delta$, namely via $g^{\text {lines }} \sim$ $g^{\text {Thomson }} \sim T_{\text {eff }}^{4}$.

Optically thick continuum. The approximation of an optically thin continuum in the wind is justified only for stars with a mass-loss rate below a critical value. For B-supergiants with mass-loss rates $\dot{M} \approx 10^{-7} \ldots 10^{-5} \mathrm{M}_{\odot} / \mathrm{yr}$, however, the Lyman continuum may become optically thick for wavelengths $\lambda \leq 911$ $\AA$. This effect has been proposed as a possible origin of equatorial disks in the winds of B[e]-supergiants for the first time by LP91. Since the Lyman continuum becomes optically thick for temperatures below $20000 \mathrm{~K}$ and mass-loss rates above a critical value, the bistability effect may become important for the wind from a rapidly rotating star with $T_{\text {eff }}=20000 \mathrm{~K}$ in the following way: Due to centrifugal acceleration, the mass-loss increases from pole to equator, and the wind should become optically thick in the Lyman continuum already at the sonic point for those colatitudes where the mass-loss exceeds a critical value. Then, the emergent flux is drastically reduced for wavelengths $\lambda \leq 911$ $\AA$, i.e. the ionizing photons, which otherwise would propagate into the wind, are blocked already in the photosphere. Thus, one should expect a two-component wind with a dense flow about the equatorial plane, where low ionization stages dominate, and a highly ionized, thin and faster wind over the poles.

In their investigation, however, LP91 performed only 1-D calculations at distinct co-latitudes of the wind and neglected the effects of non-radial forces and gravity darkening. In order to check the validity of this simplified model we have simulated a wind from a rapidly rotating B-supergiant with $T_{\text {eff }}=20000 \mathrm{~K}$. $\tau_{\mathrm{Ly}}$ is a very sensitive function of temperature, and its behaviour has not been entirely investigated so far. Therefore, we restrict ourselves to a wind model with a uniformly bright stellar surface. Fig. 3 displays the density structure and the radial velocity field for such a model. Indeed, the two-component solution proposed by Lamers \& Pauldrach is confirmed, whereby the dense flow in the equatorial plane is markedly slower than the wind over the poles. The ratio of the local mass-loss rates $\dot{M}\left(90^{\circ}\right) / \dot{M}\left(0^{\circ}\right)$ with values up to $\sim 70$ (at the stellar surface) shows good qualitative agreement with the estimates given by Zickgraf et al. (1986) and LP91.

To illustrate the differences to the wind model with an optically thin continuum, Fig. 4 (top) displays the contribution from single elements to the line force. Again, iron is the most important element. In the equatorial plane, however, $N_{\text {eff }}$ markedly exceeds the values over the pole. This arises from FeII being the major ionization stage, since the ionizing flux is drastically reduced for $\lambda \leq 911$ $\AA$, and the ionization potential of the transition from Fell to FelII corresponds to $766.1 \AA$. Over the pole, in contrast, Fell contributes most to the line force, as shown in Fig. 4 (bottom, left). 

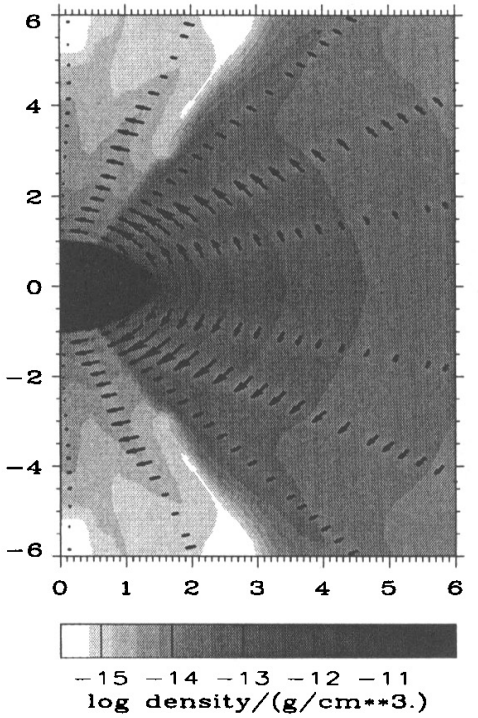

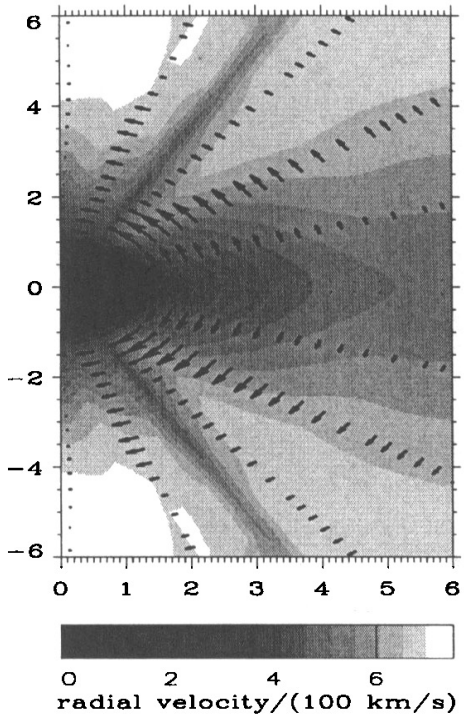

radial velocity/(100 $\mathrm{km} / \mathrm{s})$

Figure 3. Density and radial velocity field of a $\mathrm{B}[\mathrm{e}]$ star wind with consistent fmps after $2 \cdot 10^{6} \mathrm{~s}$. Uniformly bright stellar surface with $T_{\text {eff }}=20000 \mathrm{~K}, M_{*}=35 \mathrm{M}_{\odot}, r_{*}=59 \mathrm{R}_{\odot}$. The arrows indicate polar velocities with a minimum value $v_{\Theta, \min } \approx-100 \mathrm{~km} \mathrm{~s}^{-1}$.
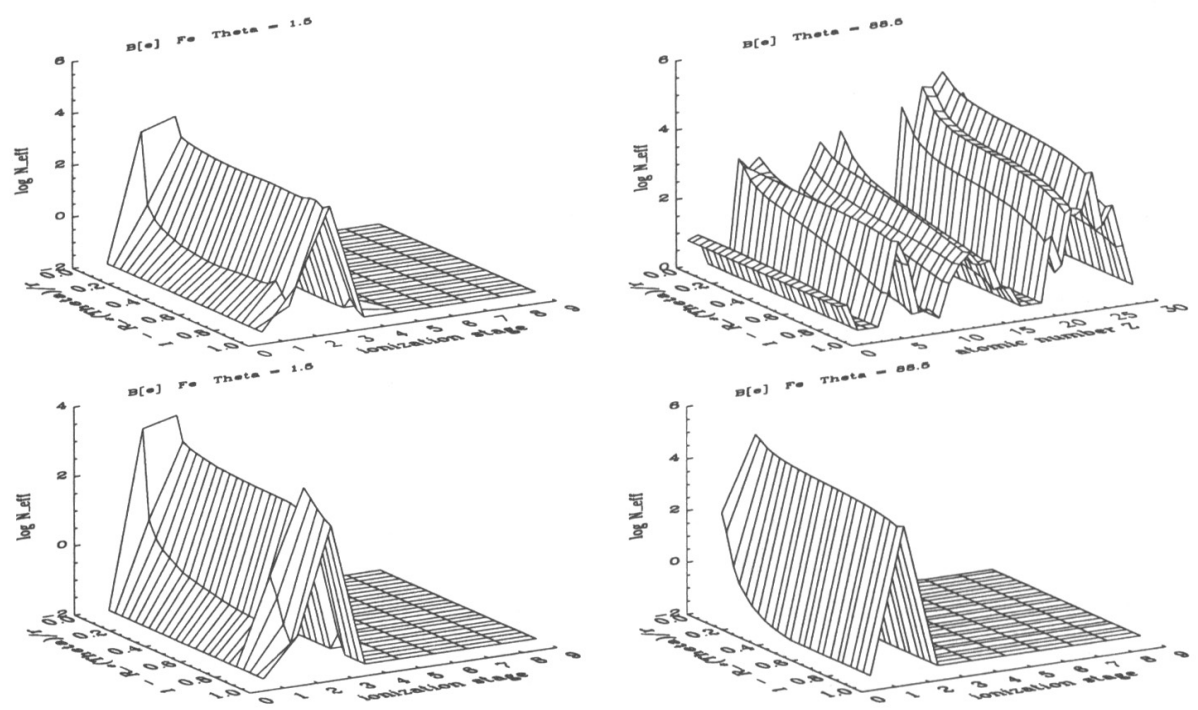

Figure 4. Top: $N_{\text {eff }}$ for the $\mathrm{B}[\mathrm{e}]$ star wind over atomic number $Z$ and radius, over the pole (left) and in the equatorial plane (right). Bottom: $N_{\text {eff }}$ for the single ionization stages of iron, over the pole (left) and in the equatorial plane (right). 


\section{Summary}

We have presented the results of radiation hydrodynamical simulations of winds from rapidly rotating early-type stars, where we have considered the dependence of the line statistics on local density and radiation temperature, thus providing values $k, \alpha, \delta$ consistent with the properties of the flow and the radiation field. In winds with an optically thin continuum, only moderate ionization gradients are present, and the global wind morphology is determined by the temperature dependence of the line force. This situation changes dramatically for winds where the continuum may become optically thick. An application to the problem of disk formation in $\mathrm{B}[\mathrm{e}]$-star winds accounting for the bistability effect shows that a two-component wind solution with a dense equatorial component and polar density contrasts $\rho_{\mathrm{p}} / \rho_{\text {eq }}$ up to $\sim 70$ may be well possible.

Future work will concentrate on the temperature dependence of the optical depth in the Lyman continuum to allow for a correct treatment of the 2-D non-LTE physics also for winds with an optically thick continuum from stars, which exhibit gravity darkening.

Acknowledgments. We would like to thank Prof. Dr. S. Owocki for engaged collaboration. This work was supported by the $D F G$ under $\mathrm{Pu} 117 / 2-1 / 2$.

\section{References}

Abbott, D.C. 1980, ApJ 242, 1183

Abbott, D.C. 1982, ApJ 263, 723

Bjorkman, J.E., Cassinelli, J.P. 1993 (BC), ApJ 409, 429

Castor, J. I., Abbott, D. C., Klein, R. I.: 1975 (CAK), ApJ 195, 157

Grinin, A. 1978, Sov. Astr. 14, 113

Lamers, H.J.G.L.M., Pauldrach, A.W.A. 1991 (LP91), A\&A 244, L5

Owocki, S.P., Cranmer, S.R., Blondin, J.M. 1994 (OCB), ApJ 424, 887

Owocki S.P., Cranmer, S.R., Gayley, K.G. 1996, ApJ 472, L115

Petrenz P., Puls, J. 1996 (PP96), A\&A 312, 195

Petrenz, P. 1999 (PP99), PhD thesis, Ludwig-Maximilians-University Munich

Petrenz, P., Puls, J. submitted to A\&A

Zickgraf, F.-J., Wolf, B., Stahl, O., Leitherer, C., Appenzeller, I. 1986, A\&A 163,119

\section{Discussion}

R. Ignace: For your B supergiant results with bistability effects, how large can $v_{\Theta}$ become, and might one expect redshifted absorption at suitable viewing inclinations?

P. Petrenz: The polar velocity component hardly exceeds $100 \mathrm{~km} \mathrm{~s}^{-1}$ and is smaller than the radial component, in most parts of the winds by two orders of magnitude. Therefore, it has no diagnostic potential for a discrimination of distinct wind models from others. 